\title{
Exploring Psychophysical Factors Influencing Visibility of Virtual Image Display
}

\author{
Shys-Fan Yang-Mao, Ming-Hui Lin, Yu-Ting Lin, Wen-Jun Zeng, and Yueh-Yi Lai \\ Industrial Technology Research Institute, Chutung, Hsinchu, Taiwan (R.O.C.) \\ \{yangmao, lmw, stevenytlin, moir,yylai\}@itri.org.tw
}

\begin{abstract}
Mixed reality (MR) or augmented reality (AR) is a very popular displaying technology in various applications. It allows the user to see the real world and virtual image simultaneously, with displaying virtual objects composited with or superimposed upon the real world. Since the usability of interactive user interface based on MR or AR relies heavily on visibility of displaying content, for virtual image display particularly. In this paper, we explore several psychophysical factors that can influence visibility on our virtual image display prototype. Factors include contrast sensitivity, transparency, color, brightness, texture gradient and ambient light. The experiment results reveal many interesting and fascinating features. The features can be the user interface design guidelines for every similar see-through near-eye display system.
\end{abstract}

Keywords: augmented reality, mixed reality, see-through near-eye display, user interface, usability, visibility.

\section{Introduction}

Augmented Reality (AR) is one part of the general area of mixed reality (MR) according to the reality-virtuality continuum, as show in Fig. 1, and it provides local virtuality [5]. Not like virtual environment (VE) or virtual reality (VR) technologies that completely immerse a user inside a synthetic environment and the user cannot see the real world around him. In contrast, AR allows the user to see the real world directly, with virtual objects composited with or superimposed upon the real world [7]. As a result, the AR technology functions by enhancing and enriching one's current perception of reality [3]. The natural and intuitional characteristics of AR increase its popularity in various applications, such as entertainment, education, industry design, personal assistance, medication, and navigation [1].

$\mathrm{AR}$ is also the technology to create a "next generation, reality-based interface" [8] and is moving from laboratories around the world into consumer markets. Current dominance head-worn AR technologies can be divided into three main types, projective AR, video AR and optical head-worn AR [1]. The optical head-worn AR (also known for see-through near-eye display) is the best choice of head-worn personal assistance for the advantages of realtime (user can see the world around him without any transmission delay) and not required for special equipment for content projection. However, see-through near-eye display has many issues: low contrast, low brightness 
for outdoor usage, low resolution, high power consuming and eye strain caused by dynamic refocus (changes from virtual objects and the real world). In order to establish a high usability user interface for see-through near-eye display, we need to overcome those issues. In this paper, we explore several psychophysical factors that can influence visibility based on our virtual image display prototype. Factors include contrast sensitivity, transparency, color, brightness, texture gradient and ambient light. We have already finished four different evaluations to explore how those factors influencing visibility. The experiment results reveal many interesting and fascinating features. The features can be the user interface design guidelines for every similar see-through near-eye display system.

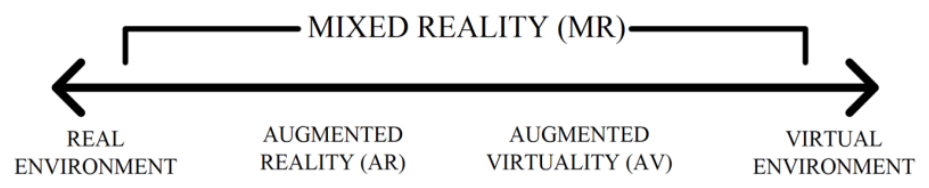

Fig. 1. Reality-virtuality continuum

\subsection{See-Through Head Mounted Display Prototype}

The prototype we used in this study is a monocular see-through near-eye virtual image display. It provides a virtual screen (10 inches in diagonal) with high-resolution ( $852 \times 480$ WVGA) that appears in front of user $(48 \mathrm{~cm})$. Since virtual screen is refracted from a very small LCD display ( 0.44 inches in diagonal) through the well-designed optical apparatus, as show in Fig. 2, the prototype has very low power consuming. The prototype also reserves extendable space for binocular applications.

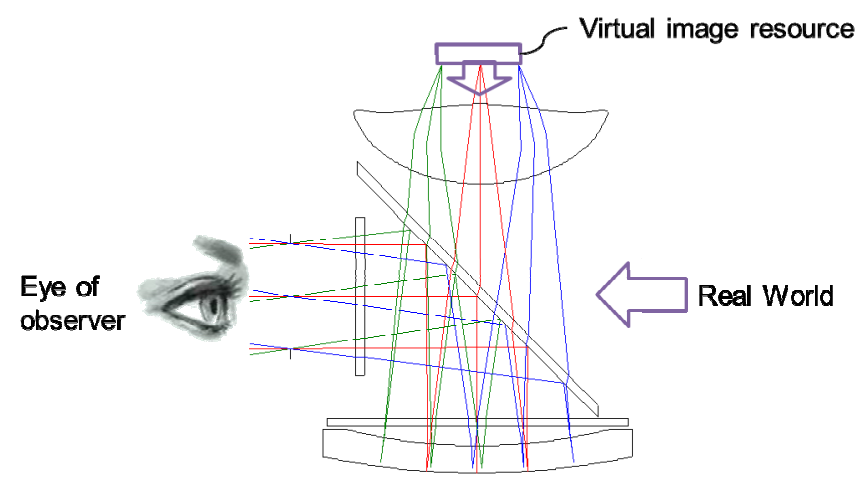

Fig. 2. Optical configuration of virtual image display prototype

\subsection{Evaluators}

In product development life cycle, the cost of changes will increase exponentially from early prototype stage to final production stage and the most important critical issues must be found and resolved as quickly and early as possible [9]. According to 
the Pareto principle, also known as the 80-20 rule, for many events, roughly $80 \%$ of the effects or problems come from $20 \%$ critical causes. The predication formula also indicates that it needs only 5 to 6 evaluators in a heuristic evaluation to find roughly $80 \%$ of usability problems [4]. Therefore, we select only 6 evaluators, with ages in 18 to 35 , participated in each evaluation in this paper. This can reduce the cost of the evaluations and preserve the effectiveness. Otherwise, we also verified that no one was color blind and all evaluators have normal or corrected-to-normal vision.

\section{Visibility Evaluations}

\subsection{Test Environment Set Up}

During the evaluations, evaluator can use one eye only to see the virtual screen and the real world through our prototype simultaneously, as show in Fig. 3. We will measure the eye dominance of evaluator use the method in prior art [2] and display test content for dominant eye only. Both eyes can see the real world. The real world is created by projection image from a high quality projector and the field of view (FOV) is limited inside and cover about half the area of projection screen. The content and background are controlled by the moderate. We also had the room totally dark $(<10$ Lux) during all the tests to eliminate all possible noise from ambient light.

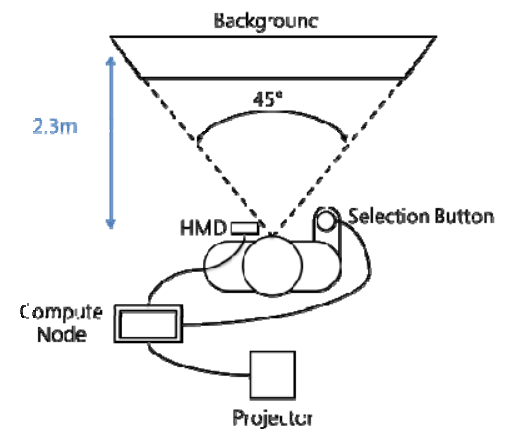

Fig. 3. Test environment set up for visibility evaluations

\subsection{Evaluations}

In this paper, we performed four different evaluations: background visibility, background related content visibility, interactive color sense and color contrast. In background visibility evaluation, we use sinusoidal line pars images as the background to simulate contrast sensitivity. Moderator will display a series of monotonic singlecolor images or white image with different transparency that cover the whole virtual screen, and project series of sinusoidal line pars images, as shown in Fig. 4, as the background. Evaluator can see the single-color virtual image and background image simultaneously. Then moderate will ask evaluator to response how clearness of the background image. The answer of clearness will scored in nine subscales which 9 is 
very clear and 1 is not clear at all. There are 9 monotonic single-color images created from the eight colors (yellow, aqua, green, orange, purple, pink, red and blue) plus white, that can be consistently named and category labeled in previous study [6]. We further generate 5 different white images with different transparency: $0 \%, 20 \%, 40 \%$, $60 \%$ and $80 \%$ as background images. In other word, white image with (R,G,B) from $(255,255,255)$ to $(51,51,51)$ in RGB color system.
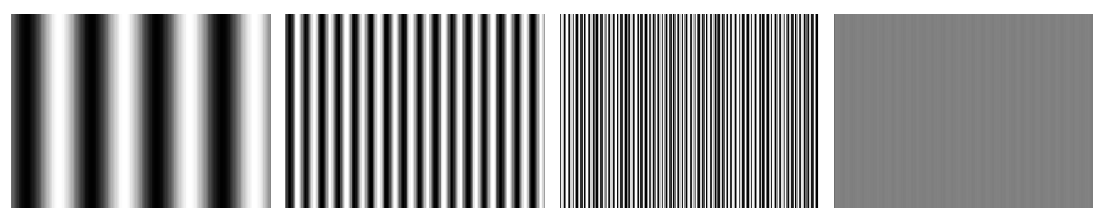

Fig. 4. Background images for background visibility evaluation
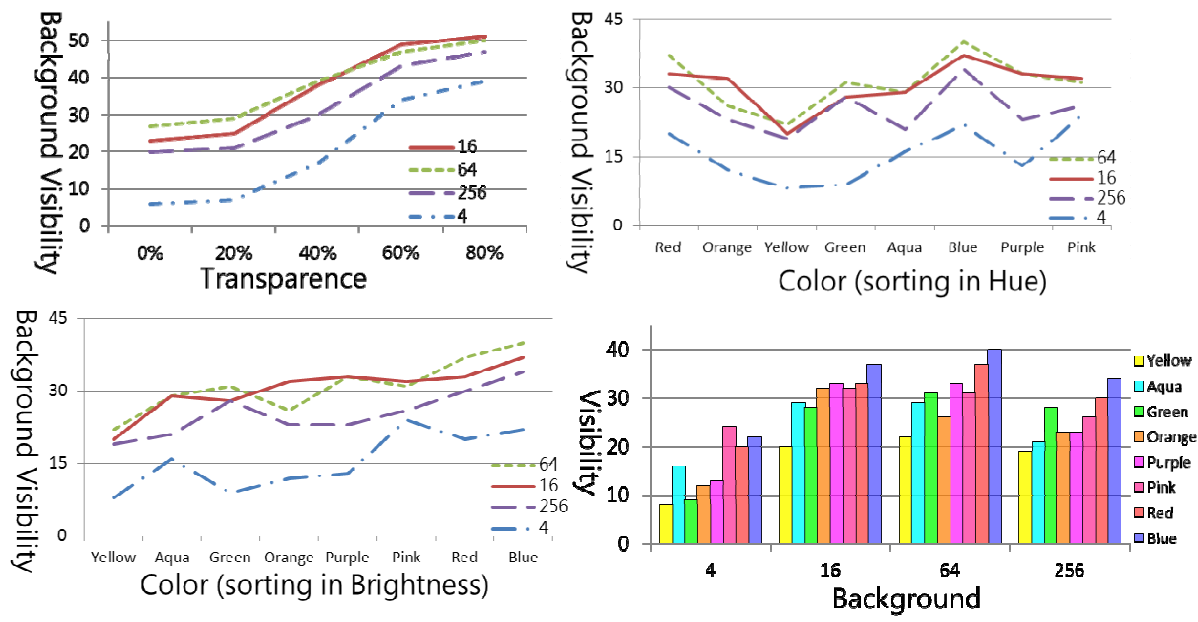

Fig. 5. Results of background visibility evaluation relative to (a) transparence (b) color sorting in Hue of HSV (c) color sorting in brightness of $\mathrm{YCbCr}(\mathrm{d})$ background images

In background related content visibility evaluation, we use random color bars image as displaying content and natural images as background to simulate the outdoor usage. Moderator will display a series of random color bars images, as shown in Fig. 6, and project a series of background images, as shown in Fig. 7, through projector. Evaluator can see the random color bars images and background image simultaneously. Then moderate will ask evaluator to report how many bars are visible on virtual screen related to current background. There are 9 color bars images and 9 bars in each content image. The width of each bar is fixed, but totally randomly in location and intensity, for example, White bars image is $(128 \pm 32,128 \pm 32,128 \pm 32)$ and Orange bars image is $(128 \pm 32,64 \pm 16,0)$ in RGB color system. Total 32 background images are collected from internet, with high resolution $(>1024 \times 768$ pixels $)$ and natural color photo only. The images contain different kinds of brightness, color and subject: such as green leafs, red flower, dark night, white cat, cars and pretty girls. 


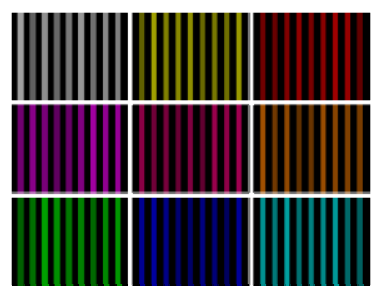

Fig. 6. Random color bar images for background related content visibility evaluation
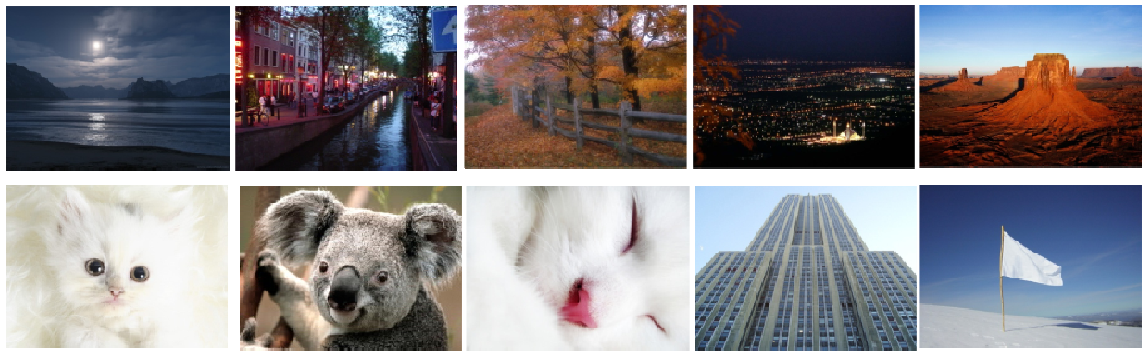

Fig. 7. Sample background images for background related content visibility evaluation

In interactive color sense evaluation, we use four different gray-level image as background and monotonic single-color images to simulate the color sensing under different ambient lights. There are three sub-tasks in this evaluation. In the first subtask, moderator display three color images (yellow, aqua, purple) with different intensity separately, and continuous showing images in increased or decreased order based on the response from evaluator. The intensity of color image will keep increase until evaluator start sense the color, and vice versa. During a constant time in test, evaluator can confirm the threshold for color sensing. In the second sub-task, thresholds for preferring color (when feel the right color with yellow, aqua and purple) sensing will be confirmed. In the third sub-task, we ask evaluators to put their finger in front of their eye and thresholds for color that affect evaluator's finger perception (when color cover or release the sense of finger) will be confirmed.

In color contrast evaluation, we use six monotonic single-color images as content and background to perform the color contrast evaluations. Six colors are red, yellow, green, aqua, blue and purple. Moderator will display one color image as background and two other colors image in virtual screen side-by-side as content. Two side-by-side square color images in virtual screen cover $1 / 3$ of FOV. Then ask evaluator to choose one preferring color that has higher color contrast from current background. There are total 120 tests in this evaluation for each evaluator. The test order is totally random.

\section{Experiment Results}

The results of background visibility evaluation reveal many interesting features, as show in Fig.5, color of content, brightness or transparency of content can change the 
visibility of background. There is a positive linear correlation in transparency of content and visibility of background image, as show in Fig. 5(a). Furthermore, the Hue and brightness of content also change the visibility of background, as show in Fig. 5(b) and 5(c), and there is a negative linear correlation in brightness of content and visibility of background. The brightness is converted from RGB color space and used in NTSC or YCbCr color space. The definition of brightness is a linear combination of $(\mathrm{R}, \mathrm{G}, \mathrm{B})$ :

$$
\mathrm{Y}=0.2126 \mathrm{R}+0.7152 \mathrm{G}+0.0722 \mathrm{~B}
$$

Color of content does not affect the contrast sensitivity of background, as show in Fig. 5(a) and Fig. 5(d). Lower (4 line pairs on sinusoidal images) or higher (256 line pairs on sinusoidal images) contrast sensitivity of background has lower visibility at every color of content.

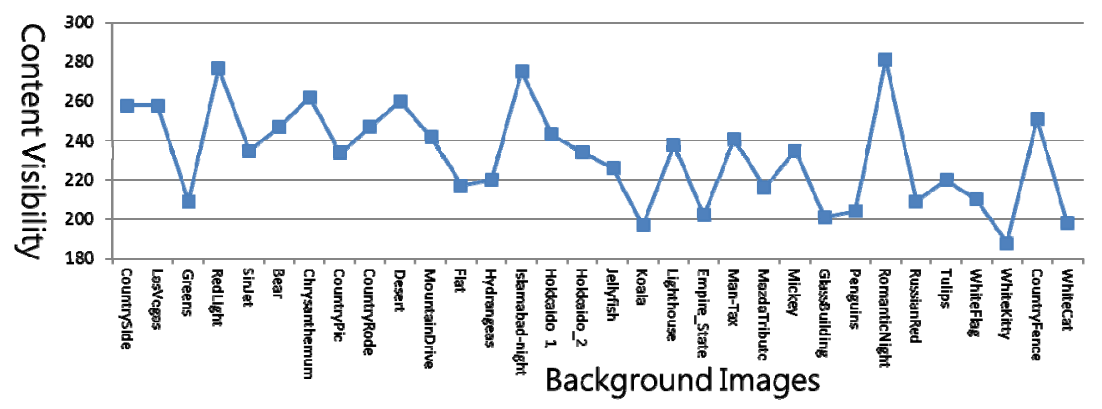

Fig. 8. Experiment results of background related content visibility evaluation for each background image

The results of background related content visibility evaluation, as show in Fig. 8 to Fig. 10, also reveal many fascinating features. Average brightness of background, color of content, color difference between content and background will change the content visibility. There is a negative linear correlation between the average brightness of background and content visibility, as show in Fig. 10(a). Five images with highest content visibility and five images with lowest visibility are shown in Fig. 7. Dark background image with far subject will increase the visibility of content. There is a positive linear correlation between the brightness of content and visibility of content, as show in Fig. 9. The random color bars images with white, yellow or green have the higher content visibility than images with blue, red or pink. Therefore, if we want to show an emergence alert or important information in virtual image display, the yellow or green will be a better choice. Color difference also changes the content visibility, as show in Fig. 10(b), and the color difference between background and content is defined as the Euclidean distance:

$$
\mathrm{D}=\left(\left(\mathrm{R} \_b-\mathrm{R} \_\mathrm{c}\right)^{\wedge} 2+\left(\mathrm{G} \_\mathrm{b}-\mathrm{G} \_\mathrm{c}\right)^{\wedge} 2+\left(\mathrm{B} \_ \text {b-B_c }\right)^{\wedge} 2\right)^{\wedge} 0.5
$$


, where background is (R_b,G_b,B_b ) and content is (R_c,G_c,B_c ) in RGB color space. There is a positive correlation between the color difference and visibility of content. Increase color difference between content and background image will also increase the visibility of content.

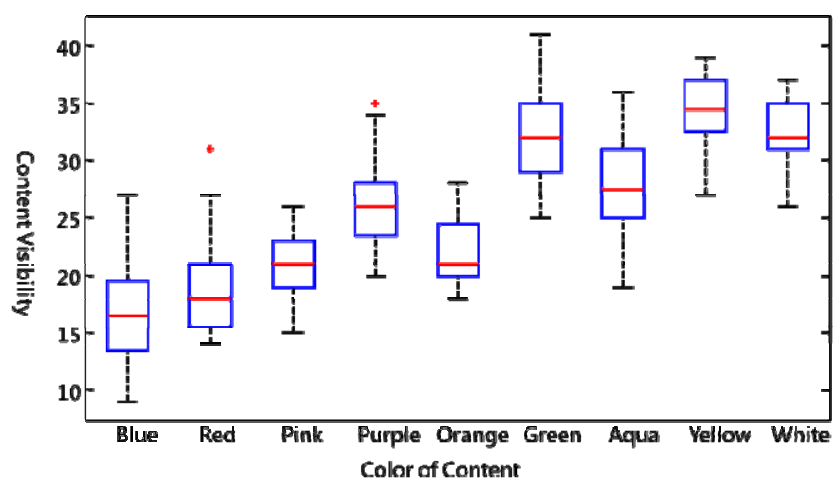

Fig. 9. Experiment results of background related content visibility evaluation for each random color bar image

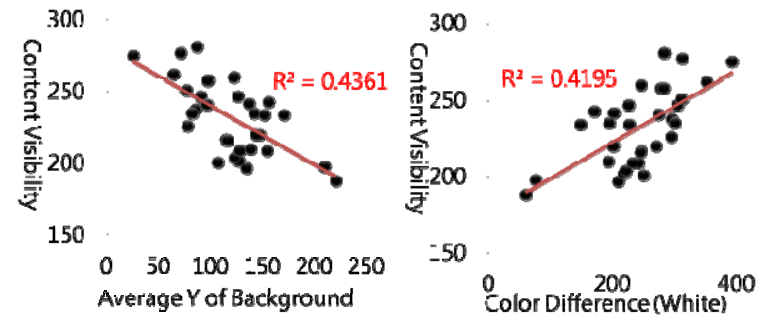

Fig. 10. Correlations between content visibility and (a) average $Y$ of background image (b) color difference between content and background image

The results of interactive color sense evaluation, as show in Fig. 11, reveal color intensity will affect the color sensing. It required higher color intensity in bright environment than that in dark. Since we can see the real world and virtual objects at the same time in AR system, it is naturally we wish to touch those virtual objects that we can see. However, the results reveal that color always affects the sense of user's finger. If we want to use the finger to interact with displaying content and without loss the sense of finger, the color intensity should dynamic decreased.

The results of color contrast evaluation, as show in Fig. 12, reveal brightness of color will affect the selection. Color with higher brightness such as yellow, green or aqua has higher selection ratio. Color blue has lowest selection ratio regardless of different background color, as show in Fig. 12(b). High brightness color can be highlight of the display content and low brightness color can balance the content. Results can be design guideline for color selection on user interface. 

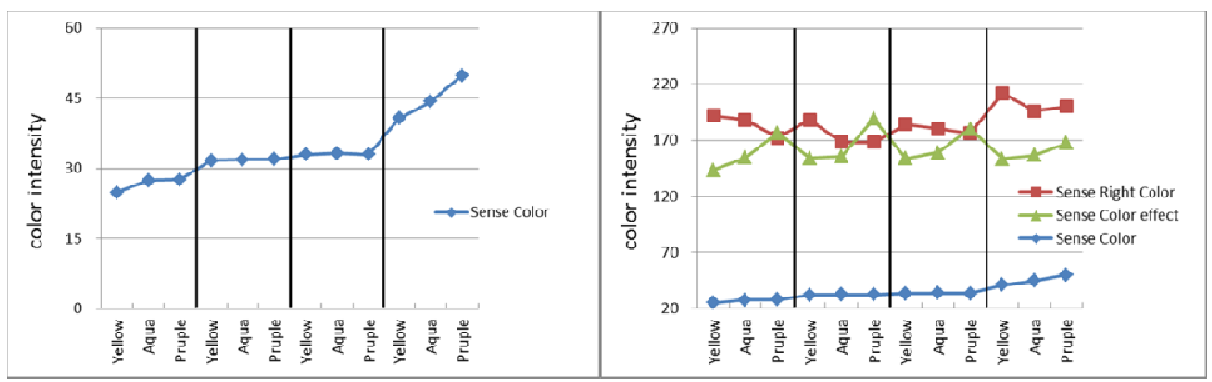

Fig. 11. Experiment results of interactive color sense evaluation

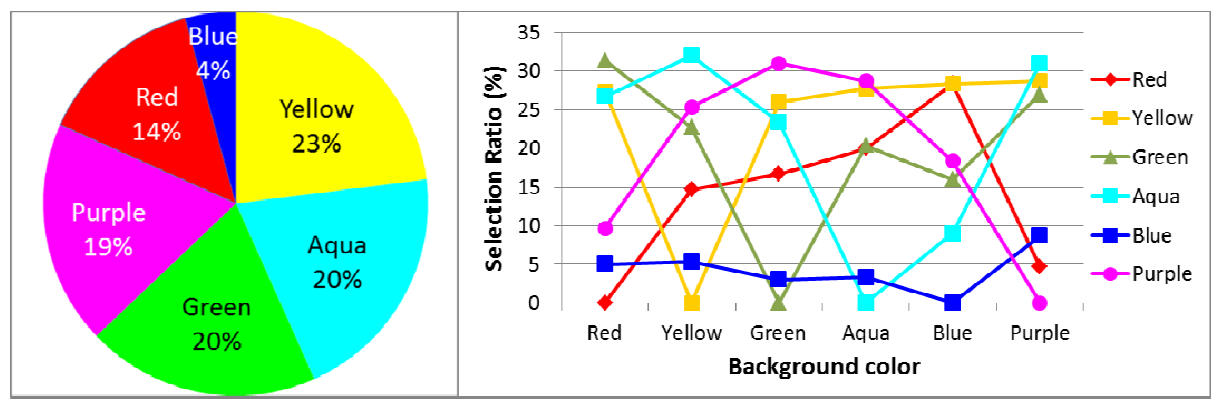

Fig. 12. Selection results of color contrast evaluation for (a) all colors and (b) different background color

\section{Conclusions and Future Works}

Augmented reality (AR) is a very popular technology in various applications. The usability of interactive user interface based on AR relies heavily on visibility of content. In this paper, we explore several psychophysical factors that can influence visibility on our virtual image display prototype. The experiment results reveal many interesting and fascinating results. Here we list several suggestions for user interface design for virtual image display based on those evaluations:

- Brightness is a good reference for color selection in user interface design.

- Red is not a good color for warning in see-through virtual image display, yellow is recommended.

- Increase color difference between content and surround will enhance the visibility of content.

- Color of content will not affect the contrast sensitivity of surround.

- Low brightness of surround will increase the visibility of content in virtual image display.

- Color of content will affect the user interaction, proper color intensity is essential and adaptive intensity is recommended.

- High brightness color can be highlight of content and low bright ness color can balance the content. 
Those suggestions can not only be the user interface design guidelines for our prototype but also for every similar see-through near-eye display system. There still remain various factors: depth perception, resolution, object motion, response time of interacting, eye strain caused by dynamic refocus, eye fatigue caused by accommodationconvergence conflict, that all might affect the usability of UI and all should be confirmed through lot of works.

\section{References}

1. van Krevelen, D.W.F., Poelman, R.: A Survey of Augmented Reality Technologies, Applications and Limitations. The International Journal of Virtual Reality 9(2), 1-20 (2010)

2. Ehrenstein, W.H., Arnold-Schulz-Gahmen, B.E., Jaschinski, W.: Eye preference within the context of binocular functions. Graefes Arch. Clin. Exp. Ophthalmol. 243(9), 926-932 (2005)

3. Graham, M., Zook, M., Boulton, A.: Augmented reality in urban places: contested content and the duplicity of code. Transactions of the Institute of British Geographers (2012), doi:10.1111/j.1475-5661.2012.00539.x

4. Nielsen, J.: Usability Engineering. Morgan Kaufmann (1993)

5. Milgram, P., Kishino, F.: A taxonomy of mixed reality visual displays. IEICE Trans. Information and Systems E77-D(12), 1321-1329 (1994)

6. Post, D.L., Greene, F.A.: Color name boundaries for equally bright stimuli on a CRT: Phase I. Society for Information Display, Digest of Technical Papers 86, 70-73 (1986)

7. Azuma, R.T.: A Survey of Augmented Reality. Presence: Teleoperators and Virtual Environments, 355-385 (1997)

8. Jebara, T., Eyster, C., Weaver, J., Starner, T., Pentland, A.: Stochasticks: Augmenting the billiards experience with probabilistic vision and wearable computers. In: First International Symposium on Wearable Computers (1997)

9. IEEE, IEEE Guide-Adoption of the Project Management Institute (PMI(R)) Standard A Guide to the Project Management Body of Knowledge (PMBOK(R) Guide), 4th edn., Project Management Institute (2011) 